\title{
sciendo
}

\section{Efficiency of SVM classifier with Word2Vec and Doc2Vec models}

\author{
Maria Mihaela TRUȘCĂ \\ Bucharest University Of Economic Studies, România \\ maria.trusca@csie.ase.ro
}

\begin{abstract}
Support Vector Machine model is one of the most intensive used text data classifiers ever since the moment of its development. However, its performance depends not only on its features but also on data preprocessing and model tuning. The main purpose of this paper is to compare the efficiency of more Support Vector Machine models using both TF-IDF approach and Word2Vec and Doc2Vec neural networks for text data representation. Besides the data vectorization process, I try to enhance the models' efficiency by identifying which kind of kernel fits better the data or if it is just better to opt for the linear case. My results prove that for the "Reuters 21578" dataset, nonlinear Support Vector Machine is more efficient when the conversion of text data into numerical attributes is realized using Word2Vec models instead of TF-IDF and Doc2Vec representations. When it is considered that data meet linear separability requirements, TF-IDF representation outperforms all other options. Surprisingly, Doc2Vec models have the lowest performance and only in terms of computational cost they provide satisfactory results. This paper proves that while Word2Vec models are truly efficient for text data representation, Doc2Vec neural networks are unable to exceed even TF-IDF index representation. This evidence contradicts the common idea according to which Doc2Vec models should provide a better insight into the training data domain than Word2Vec models and certainly than the TF-IDF index.
\end{abstract}

Keywords: Textual Analysis, Support Vector Machine, Word2Vec, Doc2Vec, TF-IDF index

\section{Introduction}

Text data classification is one of the basic NLP (Natural Language Preprocessing) operations and its applicability includes tasks like text summarization, sentiment analysis and email categorization. Text classification has got a more important role, especially in the last years because of the raising of unstructured text data available on emails, blogs or web pages that need to be classified in order to facilitate information extraction.

Generally, text data classification tasks can be tackled using either "bag of words" /manual approach or statistical models. The first solution is the simplest and the most intuitive due to the fact that a document is assigned to a class using just the most frequent words. The second approach is more elaborated and needs some training data for capturing the class features. Even if the major drawbacks of the second approach models are tied to the training set size and sometimes to the domain field data, they are by far more robust and efficient than the basic manual approach. Currently, statistical classification is developed using a large toolkit of supervised classification models like linear classifiers (Bayesian classifier, logit model), SVM (support vector machine), kNN (k-nearest-neighbor), decision trees and neural networks. Even if each of these models has their pros and cons and their performance usually depends a lot on the text data used for training I consider that one of the best of them is SVM model due to its flexibility (SVM model can handle both linear and nonlinear data) and its robustness against errors.

In this paper, I intend to present some SVM model configurations for performance 
improvement. In the first place, I try to identify the most efficient data representation method for my corpus in terms of performance and complexity cost. For this purpose, I compare simple TF-IDF (term frequency - inverse document frequency) index document representation with the one generated by Word2Vec and Doc2Vec neural networks. It is already well known that deep learning is a field that in the last years has got a serious reviving and its concepts are used by a lot of decision-makers to solve their current tasks or to enhance the quality of some older algorithms. So, my goal is to choose the best data representation approach given the benchmark dataset "Reuters 21578" and to check if indeed neural networks are able to perform better than other sample heuristics like the TFIDF index. In my attempt to identify the best vectorization process I also try to find out if my data is linearly separable and in the case of nonlinearity I try to identify the most suitable kernel taking into account radial basis and polynomial functions.

The remainder of the paper is organized as follows. The next section presents the literature review relevant to the paper's topic. The third and fourth sections provide an overview of the SVM model and vectorization processes (Word2Vec and Doc2Vec neural networks). The fifth section is dedicated to experiments and the last section presents a short conclusion of my paper.

\section{Literature review}

SVM model, one of the most common classifiers, has been extensively applied for textual data due to its robustness, low processing time and high performance that outperform other popular classification models like Naïve Bayes or kNN (Hmeidi et al., 2008). The SVM model's core implementation consists of decision function development and it is especially recommended when data sparsity is high and feature space is large (Manning et al., 2009). However, using some good preprocessing techniques the model could provide satisfactory results even for small training sets (Colas \& Brazdil, 2006).

The SVM model can handle both linear and non-linear classification tasks. The last task is achieved by changing the dimensionality of the input feature space using kernel functions. So, the SVM's efficiency depends on the kernel's choice. The most common used kernel is RBF (radial basis function) but there are a lot of others available options that can provide even better results like polynomial function kernel (Boujelbene et al., 2010), Pearson function based universal kernel (PUK) (Trive \& Dey, 2013) or even a mixture of them (Smiths \& Jordaan, 2002).

Like any other classification algorithm, the SVM model works with numerical data meaning that all training set documents should pass through the vectorization process. While the simplest bag-of-words approach provides poor results its improved version, the TF-IDF method manages to deal with stop words and assigns to each word a relevance score (Yuntao et al., 2005). Still, the two methods cannot identify semantic features and ignore the words ordering in phrases. In this case, an approach to deal with both these problems would be to apply some of the state-of-the-art models for documents numerical representation like Word2Vec (Lilleberg et al., 2015; Şahin, 2017) and Doc2Vec (Chen et al., 2015). The two models are computed in a similar way but their advantages are a bit different. While Word2Vec models generate word embeddings that can be easily applied to different documents domains, Doc2Vec models are more tied to the training set domain providing a better insight of it. Even if Word2Vec models are more flexible as regards the training domain, Doc2vec models are more advantageous when document size is large (Lau \& 
Baldwin, 2016). However, it is supposed that both neural networks outperform traditional TF-IDF representation (Bhatt et al., 2016).

\section{Overview of Support Vector Machine}

SVM, one of the most popular classification models, is widely applied for text processing, images categorization or hand-written recognition. The standard SVM model was proposed in 1993 based on the theory developed three decades earlier by Vapnik and Chervonenkis. Basically, the SVM model's aim is to classify objects into two major classes using a classifier defined for linear case in the following way:

$$
f(x)=\operatorname{sign}\left(w^{t} x+b\right)
$$

Where $w$ indicates a weight vector used for defining decision boundary and $\mathrm{b}$ is a bias term. If $\mathrm{f} f(x)=1$ than $x$ belongs to the first class and if the function value is -1 the object belongs to the second class; $f(x)=0$ indicates optimal hyperplane (in Manning et al., 2009, p. 319-349). Unlikely other classification models (eg. Naïve Bayes) that only define conceptually notion of decision boundary, the SVM model tries to identify it by maximizing the scaled distance $r_{i}$ between it and any other dot in the features space. The closest dots to the decision boundary are called support vectors. Conventionally, it is considered that the functional margin $y_{i}\left(w^{t} x+b\right)$ or unscaled distance between class elements and optimal hyperplane is at least 1 ( 1 for all support vectors); $r_{i}$ is then:

$$
r_{i}=\frac{y_{i}\left(w^{t} x+b\right)}{|w|} \geq \frac{1}{|w|}
$$

Knowing that geometric margin represents the distance between two support vectors of two different classes and $\mathrm{N}$ represents training set volume, the optimal hyperplane is defined by the parameters $(w, b)$ that solve the following optimization problem:

$$
\left\{\begin{array}{c}
\max \frac{2}{|w|} \\
y_{i}\left(w^{t} x+b\right) \geq 1
\end{array}\right.
$$

More commonly, the above optimization problem is turned into its equivalent dual problem:

$$
\left\{\begin{array}{c}
\max \sum_{i=0}^{N} \alpha_{i} \sum_{i=1}^{N} \alpha_{i} \alpha_{j} y_{i} y_{j} x_{i}^{t} x_{j} \\
\sum_{i=1}^{M} \alpha_{i} y_{i} \geq 1 \\
\alpha_{1}, \ldots, \alpha_{N} \geq 0
\end{array}\right.
$$
vector)

The parameters set $(w, \mathrm{~b})$ is then $\left(\sum_{i=1}^{N} \alpha_{i} y_{i} x_{i}, y_{i}-w^{t} x_{i}\right)$ (where $x_{i}$ is not a support

SVM presented so far works only for linearly separable classes. However, most of the classification problems do not allow linear decision boundaries meaning that it is suitable to address them using a non-linear SVM model. The most common non-linear SVM approaches use kernel trick that maps the n-dimensional feature space to a m-dimensional space, where $\mathrm{m}$ is larger than $\mathrm{n}$. The input mapping assumes generation of a new function : $x \rightarrow \phi(x)$ used for kernel function computation: $K\left(x_{i} x_{j}\right)=\phi\left(x_{i}\right)^{t} \phi\left(x_{j}\right)$.

The classification function turns into: 


$$
f(x)=\operatorname{sign}\left(\sum_{i=1}^{N} \alpha_{i} y_{i} K\left(x_{i} x\right)+b\right)
$$

There are two main approaches for dealing with multi-class SVM: one-versus-rest ("ovr") and one-versus-one ("ovo"). For n available classes, "ovr" and "ovo" approaches generate $n(n-1)$ and $n(n-1) / 2$ training sets, respectively. Usually, the results of the two methods converge.

\section{Vectorization process}

The majority of classifiers works with numerical data meaning that text data need to pass through a vectorization step. However, before this step, it is important to remove all unnecessary information like stop words, numbers, punctuation signs or hyperlinks. Stop words are usually eliminated using predefined word vectors or using some metrics for identification of irrelevant words like TF-IDF, TF (term frequency) or WF-IDF (weighting term frequency - inverse document frequency). Other common preprocessing operations are stemming and lemmatization. Both operations assume cutting words' prefixes and/or suffixes and replacing of all family words to a single one. The difference consists in the fact that lemmatization process is more elaborate and try to replace words to another dictionary word while stemming operation just changes the words with a root word (Jivani, 2011). The stemming process is more often used than lemmatization because it requires fewer resources. The most common stemmers are Porter's algorithm (Porter, 1980) and Krovetz's model. The main disadvantage of stemmer algorithms is that usually they are developed just for English text data and only sometimes for other popular languages like Chinese, Spanish or French

After text data cleaning, the vectorization process converts it into numerical inputs for statistical classifiers. Common approaches for data representation like bag-of-words and TF-IDF even if are easy to compute have some downsides: ignore words ordering in sentences and do not capture semantic features. These disadvantages could be tackled by implementing Word2Vec and Doc2Vec neural networks whose weight matrices are used for text data representation.

\section{Word2Vec}

Word2Vec word embeddings are trained using neural networks built for word prediction. Depending on the number of predicted words, there are two types of neural networks: continuous-bag-of-words (CBOW) model predicts the next word giving a set of words as input (approach similar to n-gram model) and skip-gram (SG) model predicts a set of words using just an input word. The structure of the two neural networks is simple: a hidden layer with a linear activation function and an output layer whose units are computed using a nonlinear activation function (Mikolov et al., 2013). The weights are trained with stochastic gradient descent method using backpropagation algorithm:

$$
\begin{gathered}
\text { Output layer weights: } v_{i j}^{(\text {new })}=v_{i j}^{(\text {old })}-\eta e_{j}^{v} h_{i} \\
\text { Hidden layer weights: } w_{i j}^{(\text {new })}=w_{i j}^{(\text {old })}-\eta e_{j}^{w} x_{i}
\end{gathered}
$$

Where: $\eta$ is the learning rate, $h_{i}$ is the i-th hidden unit, $e_{j}^{v}=\partial L / \partial u_{j}, e_{j}^{w}=\partial L / \partial h_{j}$, L is the loss function and $u_{j}$ is a score computed as a dot product between $h$ and the j-th column of matrix V. W and V are the weight matrices of input and hidden layers respectively. 
After the model is trained, embeddings of each word are represented by the corresponding column, $\mathrm{w}_{\mathrm{j}}$ of weight matrix $\mathrm{W}$. Text data numerical representation can be obtained by word vectors concatenation or aggregation. In the first case corpus documents are represented in a bi-dimensional space and in the second case are represented just by vectors (Rong, 2016).

\section{Doc2Vec}

Just like Word2Vec, Doc2Vec model predicts words by training two neural networks: Distributed Memory (DM) and Distributed Bag of Words (DBOW), whose architecture is similar to the one depicted above for Word2Vec models. DM neural network is similar to the CBOW model and predicts a word giving a set of words and a document token. Words and documents weights are kept in matrices $\mathrm{W}$ and $\mathrm{D}$ respectively and for prediction task, the document token vector (a column of D matrix) is either averaged or concatenated with the word vectors (columns of W matrix). DBOW neural network is similar to SG model in terms of prediction of multiple words using a unique input but unlikely SG model the input is not a word but a document token. After training Doc2Vec neural networks, columns of D matrix could be used directly for documents representation such that any other extra operation is not necessary (Quoc \& Mikolov, 2014).

\section{Results}

SVM model performance based on vectorization techniques is presented using "Reuters 21578", a benchmark dataset composed of 10788 Reuters publications separated in 90 classes. Besides the linear case, SVM's nonlinearity is checked using RBF and polynomial kernels. The SVM model is trained using TF-IDF, Word2Vec (CBOW and SG) and Doc2Vec (DBOW and DM) textual data representations. In the case of Word2Vec documents vectors are computed by summation of all their word embeddings. As regards Doc2Vec, document vectors are created easier so that no other operation is necessary except of DBOW and DM neural networks generation. In the case of DM neural network, the document token vector can be concatenated or averaged with word vectors resulting in two models: Distributed Memory Mean (DMM) and Distributed Memory Concatenated (DMC). Both versions are presented below. All models are run using Python 3.6.

Performance is checked out using precision, recall and F-measure metrics. Precision and recall show the percent of correct predicted elements for each predicted class and real class, respectively. F-score represents a good summarization of both two measures and an efficiency index:

$$
P=\frac{T P}{T P+F P} \quad R=\frac{T P}{T P+F N} \quad F=\frac{2 P R}{P+R}
$$

Where TP, FP and FN indicates the number of true positive, false positive and false negative facts, respectively. The micro-averaging approach considers that all classes are the same and the final computation is obtained by averaging all classes precision and recall metrics. The macro-averaging approach assumes that TP, FP and FP are computed independently for each class and then precision and recall are generated at the corpus level. Giving for example two classes micro-averaging and macro-averaging metrics are computed in the following way:

$$
P_{\text {micro-avg }}=\frac{T P_{1}+T P_{2}}{T P_{1}+T P_{2}+F P_{1}+F P_{2}}, \quad P_{\text {macro-avg }}=\frac{P_{1}+P_{2}}{2}
$$




$$
\begin{aligned}
R_{\text {micro-avg }}= & \frac{T P_{1}+T P_{2}}{T P_{1}+T P_{2}+F N_{1}+F N_{2}}, \quad R_{\text {macro-avg }}=\frac{R_{1}+R_{2}}{2} \\
& F_{\text {micro-avg }}=\frac{2 * P_{\text {micro-avg }} * R_{\text {micro-avg }}}{P_{\text {micro-avg }}+R_{\text {micro-avg }}} \\
& F_{\text {macro-avg }}=\frac{2 * P_{\text {macro-avg }} * R_{\text {macro-avg }}}{P_{\text {macro-avg }}+R_{\text {macro-avg }}}
\end{aligned}
$$

Analyzing all SVM models, it can be noticed that linear and non-linear SVM performance are similar and Word2Vec models outperform not only TF-IDF representation but also Doc2Vec neural networks. In terms of kernel choosing for nonlinearity handling, it can be noticed that the polynomial kernel provides slightly better results than the RBF kernel.

Table 1. Linear SVM

\begin{tabular}{|l|l|l|l|l|l|l|}
\hline & Precision & Recall & F-measure \\
\cline { 2 - 7 } & Micro-Avg & Macro-Avg & Micro-Avg & Macro-Avg & Micro-Avg & Macro-Avg \\
\hline TF-IDF & 0.98 & 0.82 & 0.93 & 0.64 & 0.95 & 0.70 \\
\hline CBOW & 0.95 & 0.98 & 0.90 & 0.94 & 0.92 & 0.96 \\
\hline SG & 0.95 & 0.98 & 0.90 & 0.95 & 0.93 & 0.96 \\
\hline DBOW & 0.93 & 0.96 & 0.79 & 0.51 & 0.91 & 0.93 \\
\hline DMC & 0.83 & 0.93 & 0.68 & 0.72 & 0.75 & 0.79 \\
\hline DMM & 0.88 & 0.95 & 0.77 & 0.83 & 0.82 & 0.88 \\
\hline
\end{tabular}

Table 2. Gaussian Kernel SVM

\begin{tabular}{|l|l|l|l|l|l|l|}
\hline & Precision & Recall & F-measure \\
\cline { 2 - 7 } & Micro-Avg & Macro-Avg & Micro-Avg & Macro-Avg & Micro-Avg & Macro-Avg \\
\hline TF-IDF & 0.99 & 0.84 & 0.94 & 0.64 & 0.96 & 0.71 \\
\hline CBOW & 1.00 & 1.00 & 0.98 & 0.87 & 0.99 & 0.92 \\
\hline SG & 0.99 & 0.97 & 0.94 & 0.72 & 0.96 & 0.81 \\
\hline DBOW & 0.97 & 0.52 & 0.78 & 0.25 & 0.87 & 0.30 \\
\hline DMC & 0.92 & 0.45 & 0.68 & 0.18 & 0.78 & 0.22 \\
\hline DMM & 0.97 & 0.76 & 0.85 & 0.43 & 0.91 & 0.53 \\
\hline
\end{tabular}

Table 3. Polynomial Kernel SVM

\begin{tabular}{|l|l|l|l|l|l|l|}
\hline & Precision & \multicolumn{3}{l|}{ Recall } & F-measure \\
\cline { 2 - 7 } & Micro-Avg & Macro-Avg & Micro-Avg & Macro-Avg & Micro-Avg & Macro-Avg \\
\hline TF-IDF & 0.99 & 0.95 & 0.97 & 0.83 & 0.98 & 0.88 \\
\hline CBOW & 1.00 & 1.00 & 0.99 & 0.99 & 0.99 & 1.00 \\
\hline SG & 1.00 & 1.00 & 0.96 & 0.94 & 0.98 & 0.97 \\
\hline DBOW & 0.99 & 0.44 & 0.61 & 0.10 & 0.76 & 0.14 \\
\hline DMC & 1.00 & 0.70 & 0.19 & 0.13 & 0.32 & 0.20 \\
\hline DMM & 0.97 & 0.99 & 0.87 & 0.77 & 0.92 & 0.86 \\
\hline
\end{tabular}

Source: Author's own research.

Besides model performance, the best model choice should also take into account computational cost. Giving the fact that there is not a noticeable difference between linear and non-linear SVM performance I choose to examine the computational cost of both models. The results are presented in Figure 1. For the case of linear SVM, Doc2Vec and TF-IDF representations have the smallest computational cost while Word2Vec CBOW neural 
network needs almost four hours to run. However, Word2Vec models have the lowest cost for the nonlinear case while TF-IDF goes far beyond the other two options and for the polynomial kernel it needs more than 5500 seconds to run while Word2Vec and Doc2Vec models do not exceed 200 secons.
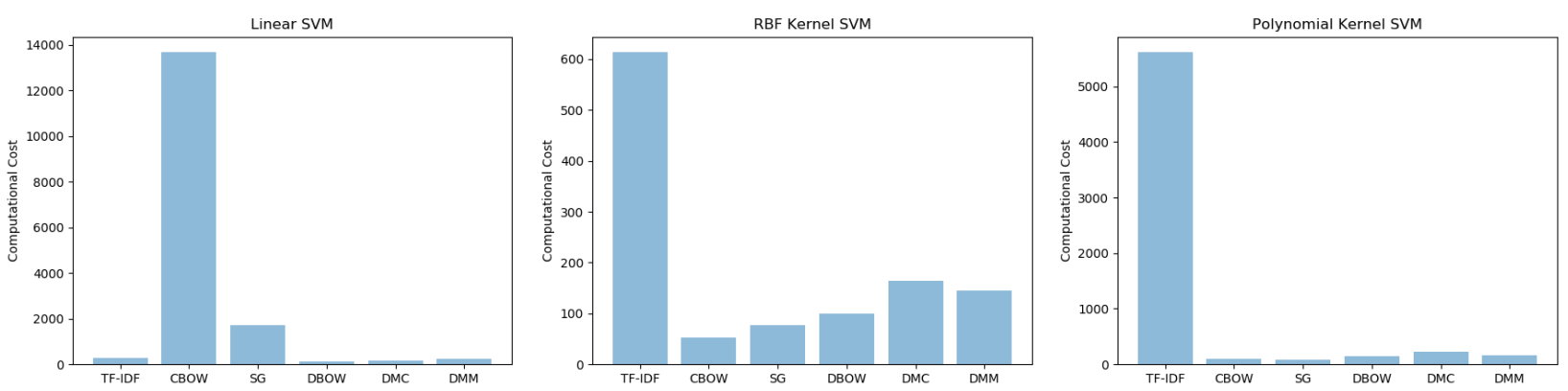

Figure 1. Computational cost (seconds)

Source: Author's own research.

Overall, for the nonlinear case, the most efficient models in terms of performance and computational cost are the ones that use Word2Vec representation with polynomial and RBF kernels. The first one guarantees the best performance while the second one has the smallest computational cost. If text data classes are considered linearly separable than it is recommended to use TF-IDF representation. As regards Doc2Vec models, they are not efficient given even if their cost is low, their performance is poor.

\section{Conclusion}

In this paper, I attempt to find the best representation for the SVM classifier choosing among TF-IDF, Word2Vec and Doc2Vec models. For each representation, both linear and nonlinear cases are evaluated. Nonlinearity is tested using two of the most efficient kernels: RBF and polynomial. Using "Reuters 21578" standard dataset I prove that Word2Vec models achieve the best performance and surprisingly TF-IDF outperforms Doc2Vec neural networks data representation. As regards computational cost, CBOW and SG neural networks again provide better results but only for the nonlinear case while Doc2Vec models and TF-IDF work better for the linear case.

Considering the good overall results of TF-IDF index representation over Doc2Vec neural networks, this paper proves that not always state-of-the-art models outperform traditional methods and sometimes a good configuration helps even the most simple approaches to provide satisfactory results.

As regards future work, I consider that it would be interesting to continue the comparison between TF-IDF index representation and other state-of-the-art models based on neural networks like global vectors for word representation (GloVe) or sentiment specific word embeddings. Even if probably these state-of-the-art models are more efficient I consider useful to explore these scenarios in order to find out new ways to improve traditional approaches like TF-IDF.

\section{References}

Bhatt, H. S., Sinha, M., \& Roy, S. (2016). Cross-domain text classification with multiple 
domains and disparate label sets. Proceedings of the 54th Annual Meeting of the Association for Computational Linguistics, (Volume 1: Long Papers) (Vol. 1, pp. 16411650).

Boujelbene, S. Z., Mezghani, D. A., \& Ellouze, N. (2010). Improving SVM by modifying kernel functions for speaker identification task. International Journal of Digital Content Technology and its Applications, 4(6), 100-105.

Chen, Y. N., Hakkani-Tür, D., \& He, X. (2015). Detecting actionable items in meetings by convolutional deep structured semantic models. 2015 IEEE Workshop on Automatic Speech Recognition and Understanding (ASRU) (pp. 375-382). IEEE.

Colas, F., \& Brazdil, P. (2006). Comparison of SVM and some older classification algorithms in text classification tasks. IFIP International Conference on Artificial Intelligence in Theory and Practice. 127, pp. 169-178. Boston: Springer.

Hmeidi, I., Hawashin, B., \& El-Qawasmeh, E. (2008). Performance of KNN and SVM classifiers on full word Arabic articles. Advanced Enaineerina Informatics, 22(1), 106-111.

Jivani, A. G. (2011). A comparative study of stemming algorithms. Int. J. Comp. Tech. Appl, 2(6), 1930-1938.

Lau, J. H., \& Baldwin, T. (2016). An empirical evaluation of doc2vec with practical insights into document embedding. arXiv preprint arXiv:1607.05368.

Lilleberg, J., Zhu, Y., \& Zhang, Y. (2015). Support vector machines and word2vec for text classification with semantic features. 2015 IEEE 14th International Conference on Cognitive Informatics \& Cognitive Computing (ICCI* CC) (pp. 136-140). Beijing: IEEE.

Manning, C. D., Raghavan, P., \& Schütze, H. (2009). An Introduction to Information Retrieval. Cambridge, England: Cambridge University Press.

Mikolov, T., Sutskever, I., Chen, S., Coraddo, G. S., \& Dean, J. (2013). Efficient estimation of word representations in vector space. Proceedings of Workshop. Scottsdale, USA.

Porter, M. (1980). An algorithm for suffix stripping. Programm, 4(3), 130-137.

Quoc, L., \& Mikolov, T. (2014). Distributed Representations of Sentences and Documents. International conference on machine learning, (pp. 1188-1196).

Rong, X. (2016). word2vec parameter learning explained. arXiv preprint arXiv:1411.2738.

Şahin, G. (2017). Turkish document classification based on Word2Vec and SVM classifier. 2017 25th Signal Processing and Communications Applications Conference (SIU) (pp. 14). Ankara: IEEE.

Smits, G. F., \& Jordaan, E. M. (2002). Improved SVM regression using mixtures of kernels. Proceedings of the 2002 International Joint Conference on Neural Networks IJCNN'02 (Cat. No. 02CH37290). 3, pp. 2785-2790. Honolulu: IEEE.

Trive, S. K., \& Dey, S. (2013). Effect of Various Kernels and Feature Selection Methods. International Journal of Computer Applications, 66(21).

Yun-tao, Z., Ling, G., \& Yong-cheng, W. (2005). An improved TF-IDF approach for text classification. Journal of Zhejiang University-Science A, 6(1), 49-55. 